\title{
BMJ Open Use and outcomes of targeted therapies in early and metastatic HER2-positive breast cancer in Australia: protocol detailing observations in a whole of population cohort
}

Benjamin Daniels, ${ }^{1}$ Sarah J Lord, ${ }^{2,3}$ Belinda E Kiely, ${ }^{3}$ Nehmat Houssami, ${ }^{4}$
Philip Haywood, ${ }^{5}$ Christine Y Lu, ${ }^{6}$ Robyn L Ward, ${ }^{7}$ Sallie-Anne Pearson, ${ }^{1}$
On behalf of the HER2 therapy observational study (HER2-OBS) investigators

\section{ABSTRACT}

To cite: Daniels B, Lord SJ, Kiely $\mathrm{BE}$, et al. Use and outcomes of targeted therapies in early and metastatic HER2-positive breast cancer in Australia: protocol detailing observations in a whole of population cohort. BMJ Open 2017;7:e014439. doi:10.1136/bmjopen-2016014439

- Prepublication history for this paper is available online. To view these files please visit the journal online (http://dx.doi.org/10.1136/ bmjopen-2016-014439).

Received 27 September 2016 Revised 22 December 2016 Accepted 29 December 2016

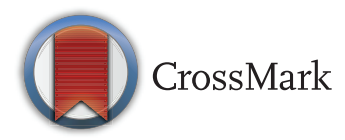

For numbered affiliations see end of article.

\section{Correspondence to} Professor Sallie-Anne Pearson; sallie.pearson@ unsw.edu.au
Background: The management of human epidermal growth factor receptor 2 (HER2)-positive breast cancer (BC) has changed dramatically with the introduction and widespread use of HER2-targeted therapies. However, there is relatively limited real-world information on patterns of use, effectiveness and safety in whole of population cohorts. The research programme detailed in this protocol will generate evidence on the prescribing patterns, safety monitoring and outcomes of patients with BC treated with HER2targeted therapies in Australia.

Methods/design: Our ongoing research programme will involve a series of retrospective cohort studies that include every patient accessing Commonwealth-funded HER2-targeted therapies for the treatment of early BC and advanced BC in Australia. At the time of writing, our cohorts consist of 11406 patients with early BC and 5631 with advanced BC who accessed trastuzumab and lapatinib between 2001 and 2014. Pertuzumab and trastuzumab emtansine were publicly funded for metastatic BC in 2015, and future data updates will include patients accessing these medicines. We will use dispensing claims for cancer and other medicines, medical service claims and demographics data for each patient accessing HER2targeted therapies to undertake this research.

Ethics and dissemination: Ethics approval has been granted by the Population Health Service Research Ethics Committee and data access approval has been granted by the Australian Department of Human Services (DHS) External Review Evaluation Committee. Our findings will be reported in peer-reviewed publications, conference presentations and policy forums. By providing detailed information on the use and outcomes associated with HER2-targeted therapies in a national cohort treated in routine clinical care, our research programme will better inform clinicians and patients about the real-world use of these treatments and will assist third-party payers to better understand the use and economic costs of these treatments.

\section{Strengths and limitations of this study}

- One of the largest and only whole-of-country HER2-positive cohorts, internationally.

- Currently up to 13 years of data observation, to be extended with future data updates.

- Linked medical services and medicines dispensing data for some patients.

- Lack of clinical measures such as Eastern Cooperative Oncology Group status and Tumour/ Nodes/Metastasis staging.

- Lack of clinical diagnoses of comorbidities, adverse events and cancer progression events.

\section{INTRODUCTION}

Amplification of the human epidermal growth factor receptor 2 (HER2) oncogene is present in $\sim 20-30 \%$ of breast cancers (BCs). ${ }^{1}$ The discovery of new and effective HER2-targeted therapies over the past 20 years has significantly improved the outcomes of patients with this aggressive BC subtype. Compared to cytotoxic chemotherapy alone, the addition of HER2-targeted therapies significantly improves response rates, disease-free survival (DFS)/ progression-free survival (PFS) and overall survival (OS) in patients with HER2-positive $\mathrm{BC}$ treated in the neoadjuvant, adjuvant or metastatic settings. ${ }^{2-12}$

While randomised clinical trials remain the gold standard for demonstrating treatment efficacy, they have some limitations as an evidence-base. The selected population enrolled in a clinical trial is not always representative of the population of 'all comers' in routine practice where patients are often older, have more extensive disease, poorer clinical status and more comorbidities. The 
sample size and duration of follow-up in clinical trials are often insufficient to detect infrequent events and to determine long-term outcomes. ${ }^{13-15}$ As a consequence, medicines can be released to market before their risk benefit profile is fully evaluated, especially when there is increasing demand for early access to potentially lifesaving medicines. Observational studies of unselected cohorts of patients are a valuable means of assessing the long-term impact of medicines and their patterns of use in routine practice. ${ }^{16} 17$

In the past decade, a number of observational studies have examined outcomes associated with HER2-targeted therapies in routine clinical practice, using data from prospective registries, hospital records and routinely collected, population-based administrative data. The heterogeneity in the available data used by these studies has driven their focus. Registry-based and hospital-based data typically include records for relatively smaller numbers of patients observed for short periods of time, but contain detailed clinico-pathological measures allowing for studies of the associations between these clinical factors and outcomes such as patterns of care following relapse, adverse events, OS and DFS/PFS. ${ }^{18-34}$

Population-based data are often maintained for purposes of reimbursement/payment and tend to have fewer clinical details, but offer much larger sample sizes across healthcare settings providing evidence more representative of general populations and allowing for better detection of rare events. To date, studies using population-based administrative data to examine the use of HER2-targeted agents in routine care have focused primarily on trastuzumab, and, to a lesser extent lapatinib, examining safety and long-term outcomes (table 1 , columns 1 and 2). Most of these studies have been conducted in North America, over a period of 5-10 observation years, in populations of up to 4000 patients. The majority of studies have focused on cardiotoxicity ${ }^{39} 4043-49$ and reported an increased risk of cardiotoxicity associated with trastuzumab treatment. A limited number of studies examined cardiac monitoring before and during trastuzumab therapy for metastatic BC $(\mathrm{MBC})$, each reporting less than half of patients underwent an assessment of cardiac function prior to initiation of therapy (range 11-38\%). ${ }^{35-37}$

Population-based study estimates of survival outcomes for women receiving HER2-targeted therapies are within the range of pivotal clinical trial estimates. Several studies reported 4-year survival rates in patients with early BC (EBC) at $\sim 90 \%{ }^{4150}$ and in patients with MBC at $41 \%{ }^{43}$ The 4 -year relapse-free survival rate in $\mathrm{MBC}$ was $76 \% .^{50}$ An Italian study found no difference in OS (HR 0.79 (95\% CI 0.50 to 1.26)) between metastatic patients previously treated with trastuzumab for EBC who are subsequently treated with trastuzumab for MBC and patients first diagnosed with $\mathrm{MBC}$ receiving trastuzumab for MBC. $^{42}$ An Australian study of patients with HER2-positive MBC estimated a median OS of 29.9 months. ${ }^{38}$
Issues, such as factors associated with use of trastuzumab, adherence to guideline-specified treatment patterns, off-label use and overall resource use, have also been examined in a number of studies. A US study found that tumour grade, ethnicity and area of residence were associated with use of trastuzumab for EBC. ${ }^{51}$ The only two studies examining lapatinib use did so in the context of quantifying resource use associated with treatment and the factors related to adherence to therapy. They found that costs did not differ between trastuzumab and lapatinib therapy, but the resource use driving costs $\mathrm{did} ;{ }^{54}$ and that prior therapy with a taxane was associated with greater discontinuation of lapatinib. ${ }^{55}$ An Australian study found that $22 \%$ of patients received trastuzumab for $\mathrm{MBC}$ with non-recommended concomitant treatment partners, and $\sim 20 \%$ (or AUD $\$ 21$ million) of trastuzumab was discarded due to regulations around unused phial portions and weekly treatment schedules. $^{35}$

Our research programme aims to provide insights into issues that clinical trials are not designed to address and contribute additional knowledge to the current evidence base on the real-world use of HER2 therapies. Specifically, we will examine real-world patterns of prescribing, sideeffect monitoring and outcomes (see table 1, column 3) using one of the largest whole-of-population cohorts of HER2-positive patients and one of the longest follow-up periods, internationally. We will:

1. Compare the real-world use and outcomes with clinical trials and guideline recommendations.

2. Determine the duration of HER2-targeted therapies and the long-term benefits and toxicities of treatment.

3. Determine the outcomes of patients receiving HER2-targeted therapies for MBC who also received HER2-targeted therapies for EBC.

4. Estimate total resources-medicines and health services-used by patients treated with HER2-targeted therapies, and factors associated with resource usage.

5. Explore the patient and treatment characteristics associated with survival.

6. Assess the impacts of policy interventions on treatment patterns and outcomes.

\section{METHODS}

\section{Study setting}

Australia maintains a publicly funded universal healthcare system entitling all citizens and permanent residents to a range of subsidised health services. This includes free treatment in public hospitals (funded jointly by the Commonwealth and State/Territory governments) and subsidised treatment in private hospitals (funded jointly by the Commonwealth and private health insurance). Outpatient services, including consultations with medical and selected healthcare professionals, are funded by the Commonwealth's Medicare Benefits Schedule (MBS). Medicines prescribed in the 
Table 1 Characteristics of published studies that use population-based administrative data and comparison with current programme

\begin{tabular}{|c|c|c|c|c|c|c|}
\hline & \multicolumn{4}{|c|}{ Published studies* } & \multicolumn{2}{|c|}{$\begin{array}{l}\text { Current } \\
\text { programme }\end{array}$} \\
\hline & EBC & Reference \# & MBC & Reference \# & $\overline{E B C}$ & MBC \\
\hline \multicolumn{7}{|l|}{ Country } \\
\hline Australia & 0 & - & 4 & $35-38$ & $\mathbf{X}$ & $\mathbf{x}$ \\
\hline Canada & 1 & 39 & 0 & - & & \\
\hline Italy & 3 & $40-42$ & 2 & 4243 & & \\
\hline USA & 10 & $44-53$ & 4 & 45465455 & & \\
\hline \multicolumn{7}{|l|}{ Observation start year } \\
\hline 1998-2000 & 4 & 45464849 & 3 & 454655 & & \\
\hline $2001-2005$ & 5 & 3944475052 & 4 & $35-38$ & & $\mathbf{X}$ \\
\hline $2006-2010$ & 5 & $40-425153$ & 3 & 424354 & $\mathbf{X}$ & \\
\hline \multicolumn{7}{|l|}{ Number of observation years } \\
\hline$<5$ & 4 & $40-4251$ & 2 & 4243 & & \\
\hline $5-10$ & 10 & $3944-505253$ & 7 & $35-38454654$ & $\mathbf{X}$ & \\
\hline$>10$ & 0 & - & 1 & 55 & & $\mathbf{X}$ \\
\hline \multicolumn{7}{|l|}{ Medicine focus } \\
\hline Trastuzumab & 14 & $39-4244-53$ & 10 & $35-38424345465455$ & $\mathbf{x}$ & \\
\hline Lapatinib & 0 & - & 1 & 55 & & \\
\hline Trastuzumab and lapatinib & 0 & - & 1 & 54 & & $\mathbf{X}$ \\
\hline \multicolumn{7}{|l|}{ HER2-positive sample size } \\
\hline$<1000$ patients & 6 & 424548495153 & 5 & 4243455455 & & \\
\hline 1000-2000 patients & 0 & - & 1 & 35 & & \\
\hline 2000-3000 patients & 6 & 404144464752 & 1 & 46 & & \\
\hline $3000-4000$ patients & 2 & 3950 & 3 & $36-38$ & & \\
\hline 5000-12000 patients & - & - & - & - & $\mathbf{x}$ & $\mathbf{X}$ \\
\hline \multicolumn{7}{|l|}{ Age } \\
\hline Patients $>65$ only & 6 & $44-474951$ & 2 & 4546 & & \\
\hline Patients of all ages & 9 & $39-4248505253$ & 8 & $35-3842435455$ & $\mathbf{X}$ & $\mathbf{X}$ \\
\hline \multicolumn{7}{|l|}{ Sex } \\
\hline Women & 12 & $39-4245-52$ & 10 & $35-38424345465455$ & & $\mathbf{X}$ \\
\hline Women and men & 2 & 4453 & 0 & - & $\mathbf{X}$ & \\
\hline \multicolumn{7}{|l|}{ Study focus } \\
\hline \multicolumn{7}{|l|}{ Treatment patterns } \\
\hline Duration of therapy & 4 & 40414753 & 5 & 3537385455 & $\mathbf{X}$ & $\mathbf{X}$ \\
\hline Schedules/dosing & 2 & 4447 & 2 & 3538 & $\mathbf{X}$ & $\mathbf{X}$ \\
\hline Concomitant cancer therapies & 13 & $39-41 \quad 44-53$ & 8 & 3537384345465455 & $\mathbf{X}$ & $\mathbf{X}$ \\
\hline Cancer therapies prior to/following HER2 therapy & 2 & 4252 & 3 & 425455 & $\mathbf{x}$ & $\mathbf{x}$ \\
\hline Non-cancer treatments & 2 & 4041 & 0 & - & $\mathbf{X}$ & $\mathbf{X}$ \\
\hline Guideline-recommended care & 2 & 4547 & 3 & 353745 & $\mathbf{x}$ & $\mathbf{x}$ \\
\hline \multicolumn{7}{|l|}{ Monitoring } \\
\hline Cardiac & 0 & - & 3 & $35-37$ & & $\mathbf{X}$ \\
\hline Other medical services & 0 & - & 2 & 5455 & & $\mathbf{X}$ \\
\hline \multicolumn{7}{|l|}{ Outcomes } \\
\hline PFS/DFS, associated factors & 3 & 415052 & 1 & 38 & & \\
\hline OS, associated factors & 5 & 4142455052 & 4 & 38424345 & $\mathbf{x}$ & $\mathbf{X}$ \\
\hline Cardiovascular events, associated factors & 7 & $39404446-49$ & 2 & 4346 & & \\
\hline
\end{tabular}

*Shih et al, Tsai et al and Negri et al include patients with EBC and MBC, and each study is included in both columns.

DFS, disease-free survival; EBC, early breast cancer; HER2, human epidermal growth factor receptor 2; MBC, metastatic breast cancer; OS, overall survival; PFS, progression-free survival.

community and some hospitals are funded by the Commonwealth's Pharmaceutical Benefits Scheme (PBS). The Australian Department of Human Services (DHS) maintains records of medicines dispensed (PBS) and medical services provided (MBS) to patients for the purpose of reimbursement.
Medicines of interest, funding and access restrictions

There are currently four publicly subsidised HER2-targeted therapies available in Australia. Medicines subsidised on the PBS are approved by the Pharmaceutical Benefits Advisory Committee (PBAC) on the basis of efficacy and cost-effectiveness. ${ }^{56}{ }^{57}$ Trastuzumab (Herceptin, 
Genentech, South San Francisco, California, USA; Hoffmann-La Roche, Basel, Switzerland) for metastatic disease was not considered to be cost-effective by PBAC, but was subsidised through a separate programme. ${ }^{58}$ From December 2001 until June 2015, the Herceptin Programme provided free access to trastuzumab for MBC. The Herceptin Programme was also administered by the DHS until its close in June 2015; since July 2015, trastuzumab for MBC has been PBS-subsidised. ${ }^{59-63}$ Trastuzumab for adjuvant and neoadjuvant treatment was listed on the PBS in October 2006 and December 2012, respectively. Lapatinib (Tykerb, GlaxoSmithKline, Research Triangle Park, North Carolina, USA) was listed on the PBS as a second-line treatment for HER2-positive MBC in May 2008. Pertuzumab (Perjeta, Genentech, South San Francisco, California, USA; Hoffmann-La Roche, Basel, Switzerland) and trastuzumab emtansine (T-DM1) (Kadcyla, Genentech, South San Francisco, California, USA; Hoffmann-La Roche, Basel, Switzerland) were listed for first-line and second-line MBC therapy, respectively, in July 2015.

To ensure that HER2-targeted agents are administered according to clinical trial evidence, the PBS places restrictions on their use. These restrictions have changed with emerging evidence and are summarised in table 2.

\section{Data sources}

Our current holdings include unit-record data on patient demographics, PBS dispensing records (all PBS-funded medicines, not just cancer medicines) and all MBS medical services records for persons treated with trastuzumab and lapatinib between January 2001 and April 2014. We will receive annual data updates. T-DM1 and pertuzumab were funded in Australia in July 2015, and patients treated with these medicines will form part of our subsequent data updates (table 3).

Australian law prevents the DHS from linking PBS to MBS records without the explicit consent of patients. ${ }^{66}$ As a result, our data holding for patients receiving PBS-funded trastuzumab (in the adjuvant or neoadjuvant settings) is currently limited to patient information and PBS dispensing history only. However, due to the Herceptin Programme arrangements (active until 2015), DHS can link PBS records and MBS records to Herceptin Programme records, separately, and supply the data, so that we can undertake the final merging of the entire data holdings. Therefore, our holdings for patients accessing trastuzumab for metastatic disease consist of patient information, PBS history (where we ascertain all other cancer therapies and other prescribed medicines), MBS history and Herceptin Programme data. We have similar data for patients who received lapatinib because access to lapatinib under the PBS required that patients progressed while receiving trastuzumab for metastatic disease, which had been only been possible through the Herceptin Programme.
Data access

Data extraction was performed by the DHS who assigned a unique scrambled ID and extracted all patient information and all dispensing records (not just HER2-targeted medicines) associated with that ID. For Herceptin Programme participants, the DHS also extracted medical services records from MBS data. Those records, with the unique ID and requested variables, were then sent to the researchers stripped of identifying information such as name and address. The researchers joined the data sets using the unique ID.

\section{Study design}

This ongoing research programme will comprise a series of retrospective cohort studies of all Australian patients with HER2-positive BC accessing publically subsidised treatment with HER2-targeted agents from 2001 to 2020.

\section{Study population}

As this is an ongoing study, the characteristics of the population will change over time. Characteristics of the study population at the date of first dispensing of HER2-targeted therapy, stratified by treatment setting, are summarised below (table 4).

In our current data holdings, there are 5631 patients who received trastuzumab and 1099 patients who received lapatinib for MBC; 11406 patients received trastuzumab in the early-stage and neoadjuvant settings. Overall, there are 1.1 million dispensing records associated with Herceptin Programme participants and 1.7 million records associated with patients with EBC and neoadjuvant patients (table 5). Herceptin Programme participants generated 2.2 million medical services claims. In total, there are 25437 total person years in the Herceptin Programme dispensing records; 59154 person years in EBC/neoadjuvant dispensing records and 27763 person years in the Herceptin Programme medical services claims (table 5).

Three thousand one hundred and thirteen of the MBC patients $(55 \%)$ and 6439 of the EBC patients $(56 \%)$ received at least one dispensing of a hormonal therapy. There were 125257 taxane dispensings and 35664 anthracycline dispensings. With a median observation time of 49.8 months (IQR 39.5-94.8) from first medicine dispensing or medical service until death or censor date (31 March 2014), 3777 of the patients treated for MBC (67\%) have died and 898 of the patients treated for EBC $(8 \%)$ have died. Reflecting the population distribution of Australia, more than half of patients in all treatment settings resided in New South Wales and Victoria and more than two-thirds of all patients lived in major cities (not shown in table 4). Among patients with $\mathrm{MBC}$, at least $81 \%$ received at least one dispensing of a pain medication; $48 \%$ received medication for the treatment of hypertension or angina; $40 \%$ received an antidepressant and $23 \%$ received an antianxiety medication. Among patients with EBC, 64\% received at least one dispensing of a pain medication; $40 \%$ received medication for hypertension or angina; 
Table 2 Access restrictions to HER2-targeted therapies in Australia

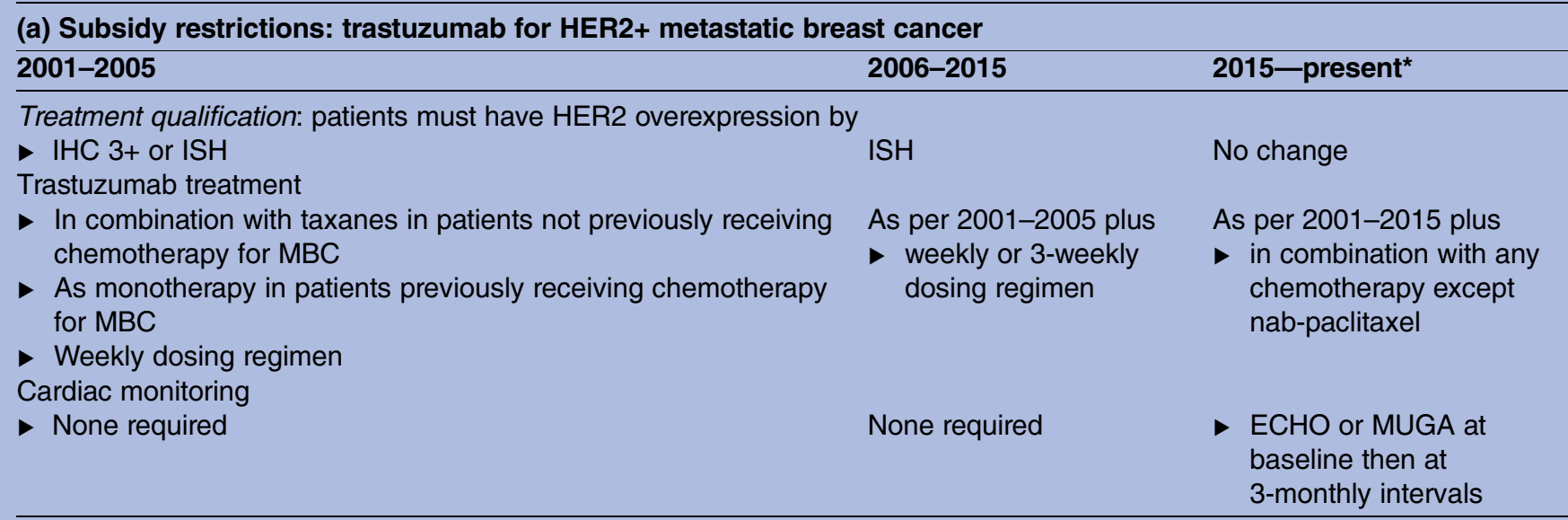

\section{(b) Subsidy restrictions: trastuzumab for HER2+ early breast cancer}

\section{6-2015}

Treatment qualification: patients must have...

- HER2 overexpression demonstrated by ISH

- Undergone surgery for breast cancer

Trastuzumab treatment

- Started in combination with chemotherapy

- Patients are eligible for 52 weeks of treatment

Cardiac monitoring

- ECHO or MUGA at baseline then at 3-monthly intervals

- Left ventricular ejection fraction (LVEF) > 45\%

- No symptomatic heart failure

(c) Subsidy restrictions: lapatinib for HER2+ metastatic breast cancer

\section{8-2010}

Treatment qualification: patients must have...

- HER2 overexpression demonstrated by ISH

- Prior taxane for $\geq 3$ cycles; or intolerance to taxane

- Disease progression while receiving trastuzumab for MBC

Lapatinib treatment

- As sole PBS-subsidised anti-HER2 treatment

- In combination with capecitabine

- Patients CANNOT receive trastuzumab subsequent to receiving lapatinib

Cardiac monitoring

- ECHO or MUGA at baseline then at discretion of clinician

\section{5-present}

No change

No change

No change

$$
\text { 2010-2015 }
$$

2015-present

No change

No change

- As sole PBS-subsidised anti-HER2 treatment

- In combination with capecitabine

- Patients $C A N$ receive trastuzumab subsequent to receiving lapatinib

No change

No change

\section{(d) Subsidy restrictions: trastuzumab for HER2+ neoadjuvant therapy}

\section{2-present}

Treatment qualification: patients must have...

- HER2 overexpression demonstrated by ISH

- Not undergone surgery for breast cancer

Trastuzumab treatment

- In combination with chemotherapy

- Patients are eligible for 52 weeks of treatment 
Table 2 Continued

\section{(d) Subsidy restrictions: trastuzumab for HER2+ neoadjuvant therapy} 2012-present

Cardiac monitoring

- ECHO or MUGA at baseline then at 3-monthly intervals

- LVEF $>45 \%$

- No symptomatic heart failure

(e) Subsidy restrictions: pertuzumab for HER2+ metastatic breast cancer 2015-present

Treatment qualification: patients must have...

- HER2 overexpression demonstrated by ISH

- WHO performance status of 0 or 1

- No prior HER2 therapy for MBC

Pertuzumab treatment

- In combination with trastuzumab and a taxane (not nab-paclitaxel)

Cardiac monitoring

- ECHO or MUGA at baseline then at 3-monthly intervals

(f) Subsidy restrictions: Trastuzumab emtansine (T-DM1) for HER2+ metastatic breast cancer

2015-2016

2016-present

Treatment qualification: patients must have

- HER2 over expression demonstrated by ISH

- WHO performance status of 0 or 1

- Progressed while receiving pertuzumab and trastuzumab for MBC or while receiving or within 6 months of completing adjuvant trastuzumab

- Not received prior treatment with lapatinib or developed an intolerance to lapatinib

T-DM1 treatment

- Treatment as monotherapy

Cardiac monitoring

- ECHO or MUGA at baseline then at 3-monthly intervals

As per 2015-2016 but

- patients may have received prior treatment with lapatinib or developed an intolerance to lapatinib

No change

${ }^{*}$ Herceptin Programme ceased and trastuzumab for MBC was listed on the PBS.

ECHO, echocardiography; HER2, human epidermal growth factor receptor 2; IHC, immunohistochemistry; ISH, in situ hybridisation;

MBC, metastatic breast cancer; MUGA, multiple gated acquisition scan; PBS, Pharmaceutical Benefits Scheme.

$35 \%$ received an antidepressant and $17 \%$ received an antianxiety medication.

Patients with MBC accessing trastuzumab had a median of 54 medical service claims per person, per year (IQR 23-106). The majority of claims relate to pathology services $(40.4 \%)$ and consultations and visits with healthcare professionals (27\%). Patients who also received lapatinib for MBC had 536370 medical service claims, with a median of 68 (27-121) per person, per year. These services followed a similar pattern to those for all trastuzumab patients.

\section{Outcomes of interest and statistical analyses}

We will use a range of pharmacoepidemiological and statistical analyses to address our aims.

\section{Patterns of use}

We will summarise the prescribing patterns of HER2-targeted therapies, including agent used, line of therapy, partnering therapy (chemotherapy, other HER2-targeted therapy and endocrine therapy) and duration of therapy.
We will report the characteristics of patients dispensed HER2-targeted therapies, including age, sex, geographical remoteness, socioeconomic status, HR status and presence of comorbidities at dispensing of HER2-targeted therapy and over time. Age, sex, geographical remoteness and socioeconomic status will be ascertained from the patient information data sets. We will define HR status using a validated proxy and define the number and nature of comorbidity from dispensing claims using the validated RxRisk index. ${ }^{67-69}$

\section{Comparison of real-world use with clinical trials and} prescribing guidelines

We will compare the duration of therapy (based on dispensing records) and survival outcomes associated with HER2-targeted therapies to those from published clinical trials; we will not undertake comparative efficacy analyses as it is prone to confounding by indication bias. We will estimate OS through Kaplan-Meier methods. We will use descriptive statistics to compare characteristics of patients treated with these medicines in the real-world setting to those treated in clinical trials. Finally, we will 
Table 3 Data holdings approved for the research programme

\begin{tabular}{|c|c|c|c|c|c|c|c|}
\hline \multirow{2}{*}{$\begin{array}{l}\text { Data set } \\
\text { First available } \\
\text { date in Australia }\end{array}$} & \multirow[t]{2}{*}{ Description } & \multicolumn{4}{|l|}{ Metastatic } & \multirow{2}{*}{$\begin{array}{l}\text { Early-stage } \\
\text { Trastuzumab } \\
2006\end{array}$} & \multirow{2}{*}{$\begin{array}{l}\text { Neoadjuvant } \\
\text { Trastuzumab } \\
2012\end{array}$} \\
\hline & & $\begin{array}{l}\text { Trastuzumab } \\
2001\end{array}$ & $\begin{array}{l}\text { Lapatinib } \\
2008 \\
\end{array}$ & $\begin{array}{l}\text { T-DM1 } \\
2015 \\
\end{array}$ & $\begin{array}{l}\text { Pertuzumab } \\
2015\end{array}$ & & \\
\hline $\begin{array}{l}\text { Patient } \\
\text { demographics }\end{array}$ & $\begin{array}{l}\text { Year of birth; sex; } \mathrm{mm} / \mathrm{yy} \text { of death; state of residence and } \\
\text { postcode of residence mapped to } \mathrm{SLA}^{*}\end{array}$ & $x$ & $x$ & $x$ & $x$ & $x$ & $x$ \\
\hline Patient weight & $\begin{array}{l}\text { Patient weight }(\mathrm{kg}) \text { at the time of Herceptin Programme } \\
\text { enrolment }\end{array}$ & $x$ & $\mathbf{X}$ & & & & \\
\hline $\begin{array}{l}\text { Treatment } \\
\text { qualification }\end{array}$ & $\begin{array}{l}\text { Patient HER2 overexpression levels and the test used to } \\
\text { ascertain levels (IHC or ISH). } \\
\text { Initial intended treatment-monotherapy or concomitant } \\
\text { treatment with taxanes }\end{array}$ & $x$ & $\mathbf{X}$ & & & & \\
\hline PBS & $\begin{array}{l}\text { All prescribed medicines reimbursed by the PBS. Variables } \\
\text { include medicine name and strength, date of prescribing, date } \\
\text { of supply, quantity supplied/pack size, the number of repeats } \\
\text { allowed with the prescription, patient copayment contribution } \\
\text { and the cost to government }\end{array}$ & $x$ & $x$ & $x$ & $\mathbf{x}$ & $x$ & $\mathbf{x}$ \\
\hline $\begin{array}{l}\text { Trastuzumab } \\
\text { supply }\end{array}$ & $\begin{array}{l}\text { Dates and phial of trastuzumab dispensed to Herceptin } \\
\text { Programme participants }\end{array}$ & $x$ & & & & & \\
\hline MBS & $\begin{array}{l}\text { All medical and allied health services. Variables includes the } \\
\text { type of service rendered-from outpatient doctor visits to } \\
\text { surgeries-the cost and benefit paid for the service, and the } \\
\text { date of service }\end{array}$ & $x$ & $\mathbf{X}$ & & & & \\
\hline
\end{tabular}


Table 4 Cohort demographic and clinical characteristics at first HER2-targeted therapy dispensing

\begin{tabular}{|c|c|c|c|}
\hline & \multicolumn{2}{|l|}{ Metastatic } & \multirow{2}{*}{$\begin{array}{l}\text { Early-stage } \\
\text { trastuzumab }\end{array}$} \\
\hline & Trastuzumab & Lapatinib & \\
\hline Patients with at least one dispensing (n) & 5631 & 1099 & 11406 \\
\hline Age, median (IQR) & $56(48-65)$ & $56(48-63)$ & $54(47-63)$ \\
\hline Weight in kilograms at first dispensing, median (IQR) & $70(60-80)$ & $70(60-81)$ & - \\
\hline HER2-positive by IHC 3+, n (\%) & $3542(62.9)$ & $585(53.2)$ & \\
\hline HER2-positive by ISH, n (\%) & 2193 (38.9) & $496(45.1)$ & \\
\hline Fact of death, $n(\%)$ & $3777(67.1)$ & $892(81.2)$ & $898(7.9)$ \\
\hline Hormone receptor positive, $n(\%)^{*}$ & $3113(55.3)$ & $617(56.1)$ & $6439(56.4)$ \\
\hline \multicolumn{4}{|l|}{ Comorbiditiest, n (\%) } \\
\hline $0-2$ & $492(8.7)$ & $44(4.0)$ & $1928(16.9)$ \\
\hline $3-4$ & $921(16.4)$ & 149 (13.6) & 3054 (26.8) \\
\hline $5-6$ & 1137 (20.2) & 244 (22.2) & 2689 (23.6) \\
\hline $7+$ & 3081 (54.7) & 662 (60.2) & 3735 (32.7) \\
\hline
\end{tabular}

Table 5 Characteristics of data holding

\begin{tabular}{lccc} 
& Metastatic & $\begin{array}{c}\text { Early-stage } \\
\text { Trastuzumab }\end{array}$ & Lapatinib \\
\cline { 2 - 3 } & Trastuzumab & 261496 & 1763268 \\
Dispensing records, total (N) & 1100594 & 8000 & 171605 \\
Dispensing records, HER2-targeted therapy (N) & 145907 & 536370 & - \\
Medical services records (N) & 2221760 & & \\
Type of medical service, overall, claims, N (\%) & & $225210(42.0)$ & - \\
Pathology & $897597(40.4)$ & $135521(25.3)$ & - \\
Attendances/consults/visits & $599277(27.0)$ & $79266(14.8)$ & - \\
Specialist & $329077(14.8)$ & $48614(9.1)$ & - \\
General practitioner & $236649(10.7)$ & $3095(0.6)$ & - \\
Enhanced primary care & $13045(0.6)$ & $2100(0.4)$ & - \\
Practice nurse & $8264(0.4)$ & $2446(0.5)$ & - \\
Other & $12242(0.6)$ & $48081(9.0)$ & - \\
Diagnostic imaging & $199411(9.0)$ & $36276(6.8)$ & - \\
Radiotherapy/nuclear medicine & $136490(6.1)$ & $91282(17.0)$
\end{tabular}

compare the real-world treatments to published treatment guidelines.

\section{Outcomes in patients who received HER2-targeted therapies} for $\mathrm{EBC}$ and $\mathrm{MBC}$

We will identify a subset of patients who initiate trastuzumab for EBC and who are subsequently trastuzumabtreated for MBC; this patient group is underrepresented in clinical trials. We will compare patient characteristics for this patient group with trastuzumab-naïve MBC patients, trastuzumab-naïve MBC patients whose first cancer medicine was trastuzumab (as a proxy for patients first diagnosed with MBC) and EBC patients who do not go on to receive trastuzumab for MBC. We will describe patterns of treatment for each of these three patient groups and use Cox proportional hazard regression to estimate differences in OS between these patient groups.

\section{Estimating total resources}

We will use multiple metrics to examine the nature and extent of resource use associated with HER2-targeted therapy. We will report on PBS, MBS and Herceptin Programme resource use overall and by service type and stratify resource use by age, treatment setting, patterns of care, socioeconomic status and remoteness. We will examine the proportion of total resource use accounted for by each service (eg, the proportion of total services accounted for by medications, imaging procedures, surgery, specialist consultations, etc). We will identify predictors of the rate of health service usage using Poisson regression or negative binomial regression, as appropriate. In all models, we will consider age at initiation of first HER2-targeted therapy, geographical remoteness, socioeconomic status, HR status and comorbidities. 


\section{Examining variations in patient response}

We will examine predictors of time-to-discontinuation and time-to-death using Kaplan-Meier curves and Cox proportional hazards models. We will ascertain date of death using the patient information data set. We will use subgroup analysis to interrogate data on patients who die during early-stage treatment or soon after its completion and those who survive for many years following initiation of HER2 therapy to determine the characteristics and patterns of treatment associated with short-term and long-term survival.

\section{Impact of policy interventions on treatment patterns and outcomes}

We will examine specific prescribing policies in Australia to determine the impact they have on treatment patterns and outcomes. For instance, during the first 2 years of its availability, prescribing lapatinib to a patient prohibited a return to trastuzumab for that same patient. We will explore the impact of policy changes using interrupted time series methodology. ${ }^{36}$

Analyses will be performed using SAS V.9.4, Stata V.13 and R V.3.2.2.

\section{Limitations}

As in any epidemiological study, we must consider the potential biases in our research. Some of the issues raised in relation to administrative database research and the conduct of pharmacoepidemiological research in Australia are described below.

\section{Medicine exposure}

Australia maintains comprehensive pharmaceutical claims data collections for prescribed medicines dispensed in community and private hospitals, but not for public hospital inpatients. The vast majority of oncology protocols are administered in the outpatient setting or to private hospital inpatients (both of which are captured in the PBS data), and we believe that the lack of public hospital inpatient dispensing data is unlikely to impact significantly on the outcomes of our analyses.

In addition, the creation of PBS records is tied to those medicines that are subsidised (in part or in full) by the government. Subsidised medicines in Australia require a patient copayment; AUD $\$ 38.30$ at the time of writing. Medicines whose cost is below this amount are not subsidised by the PBS and are not recorded in the PBS data. Therefore, the record of patients' PBS medicine use may be incomplete, limiting the scope of some analyses. ${ }^{57}$ We do, however, have information on all PBS medicines, including their total costs over time and the capacity to identify patients for whom we may not have all PBS dispensings (using their entitlement category). We will restrict some of our analyses to persons with complete PBS medicines ascertainment. Importantly, the vast majority of cancer medicines are above the copayment threshold. ${ }^{70}$ Furthermore, from July 2012 under copayment, medicines were recorded in PBS data and these records will be a part of future data updates.

\section{Diagnosis, outcome and covariate misclassification}

Health administrative data sets lack detailed clinical information, and we need to assess the impact of misclassifying diagnoses and outcomes of interest. Owing to the structure of the data sets, we know that all patients with MBC appear in Herceptin Programme data sets. For patients with early BC, between 1 October 2006 and 30 November 2012, all dispensings of trastuzumab represent adjuvant therapy, as this was the only PBS-funded indication during this time. As noted earlier, the Herceptin Programme was phased out in 2015 and trastuzumab for MBC listed on the PBS, meaning that from late 2015 trastuzumab dispensings across all treatment settings form part of the PBS data; based on our existing current data holdings, we will not be able to distinguish between trastuzumab supplied for metastatic and early-stage disease from late 2015. Similarly, among patients with early BC from 1 December 2012, we are unable to determine which dispensings represent adjuvant or neoadjuvant therapy.

To address this issue, we will obtain dispensing authority codes. Authority codes are generated when the prescribing doctor gains approval to administer an authority-required medicine (such as all HER2-targeted therapies) for a particular indication, and they will allow us to delineate between medicines dispensed across the different settings.

The data also lack certain important covariates, including comorbidities, Eastern Cooperative Oncology Group (ECOG) status and Tumour/Nodes/Metastasis (TNM) staging. Identifying adverse events, such as cardiotoxic events, is difficult without detailed clinical information or hospital admissions codes. Additional, external data sets may be used to examine these issues, but we will not attempt these analyses with our current data holdings.

We previously attempted to validate a proxy for disease progression using dispensing claims, but demonstrated a sensitivity of $74 \%$, specificity of $88 \%$ and positive predictive value of $61 \% .^{71}$ As such, we do not currently have the capacity to accurately estimate time to progression or PFS using dispensing claims alone. This will limit the scope of outcomes research in the patients with early-stage disease; at present, the main contributions based on our available data are likely to lie in the metastatic setting.

\section{Ethics}

At the time of writing, we have ethical approval for annual data updates until 2020.

The data for the research programme are released without individual consent. The use and disclosure of Commonwealth data are governed under the Privacy Act 1988. Information Privacy Principle (IPP) 2 under the Privacy Act 1988 (Commonwealth) provides that personal information should not be used or disclosed for any purpose other than the primary purpose of the collection. 
We sought approval to use the data for a secondary purpose, that of research involving data linkage.

- Under IPP2.1(d) use or disclosure for another purpose is permitted if (1) it is necessary for research and it is impracticable to gain consent and (2) the use is in accordance with the section 95A guidelines (which provide a process to resolve the conflict that may arise between the public interest in privacy and the public interest in medical research).

We applied for these exemptions to the current research programme. Individual consent for the release of data has been waived because:

- It is not possible or practical to obtain consent because of the large study population $(>15000$ patients), and a large proportion of patients were likely to be deceased.

- Obtaining consent would prejudice the scientific value of the research due to the high participation rates required for unbiased samples (at least 90\%) and the Australian evidence about the sociodemographic differences between participants who consent to data linkage research and those who do not. ${ }^{72} 73$

- The public interest in the research outweighs the public interest in privacy protection, as we know little about the way in which HER2-blockade medicines are used in the real-world marketplace.

\section{DISSEMINATION PLAN}

We will consult clinicians, policymakers and consumers where appropriate for guidance in interpreting and disseminating our results. The outcomes of this research will be submitted to international peer-reviewed journals; in particular oncology, general medical and pharmacoepidemiology journals. We will also present our findings at national and international oncology and pharmacoepidemiology conferences. We will communicate study outcomes to relevant professional cancer/oncology societies, such as the Clinical Oncology Society of Australia and the Medical Oncology Group of Australia, and policy groups such as the Pharmaceutical Benefits Advisory Committee and NPSMedicinewise. We will also develop lay summaries of research findings as needed.

In accordance with our DHS data agreement, we will submit all data that will be communicated in the public domain to the DHS for review and approval. Authorship will be based on the International Committee of Medical Journal Editors guidelines. ${ }^{74}$ Outcomes will also be posted on the University of New South Wales web page of the lead investigator and the Centre for Big Data Research in Health website. Direct access to the data and analytical files to other individuals or authorities is not permitted without the express permission of the approving human research ethics committees and data custodians.

\section{DISCUSSION}

The programme of research outlined in this protocol will provide valuable evidence of the real-world, clinical use and outcomes of HER2-targeted therapies. The unique funding structure of these medicines in Australia has created one of the largest and only whole-of-country, HER2-targeted therapies data sets in the world. Observational studies of the kind described in this protocol are particularly important, given many of the patients treated in routine practice would not meet typical clinical trial inclusion criteria. The existing observational research has highlighted the use of trastuzumab in populations significantly different from those in the clinical trials; at present, there is limited information on the real-world use of lapatinib and no studies addressing T-DM1 or pertuzumab.

The strengths of this programme lie in the use of best practice methods to examine patterns of use and longterm outcomes associated with HER2-targeted therapy; this is particularly important for patients with survival times longer than the typical clinical trial follow-up period. Given these data come from a single payer and are national in scope, loss to follow-up is likely to be much lower than observational studies conducted in countries where health service provision and insurance is more fragmented. Owing to the whole-of-population nature of the data, our findings are likely to be highly generalisable and provide opportunities to extend knowledge on the population impact of HER2-targeted therapy.

\section{Author affiliations}

${ }^{1}$ Medicines Policy Research Unit, Centre for Big Data Research in Health, UNSW, Sydney, New South Wales, Australia

${ }^{2}$ School of Medicine, University of Notre Dame Australia, Sydney, New South Wales, Australia

${ }^{3}$ NHMRC Clinical Trials Centre, University of Sydney, Sydney, New South Wales, Australia

${ }^{4}$ Sydney School of Public Health, Sydney Medical School, University of Sydney, Sydney, New South Wales, Australia

${ }^{5}$ Centre for Health Economics Research and Evaluation, University of Technology Sydney, Sydney, New South Wales, Australia

${ }^{6}$ Department of Population Medicine, Harvard Medical School and Harvard

Pilgrim Health Care Institute, Boston, Massachusetts, USA

${ }^{7}$ University of Queensland, Brisbane, Queensland, Australia

Acknowledgements The authors thank the Department of Human Services for providing the data for this research.

Collaborators HER2 therapy observational study (HER2-OBS) investigators.

Contributors BD, SJL, BEK, NH, PH, CYL, RLW and S-AP conceived of the study protocol. BD, RLW and S-AP contributed to the acquisition of the data. $\mathrm{BD}$ conducted the literature search and performed the data analyses. BD, SJL, $\mathrm{BEK}, \mathrm{NH}, \mathrm{PH}, \mathrm{CYL}, \mathrm{RLW}$ and S-AP contributed to the design of the work and interpretation of the data.

Funding This research is supported, in part, by a Cancer Australia Priority Driven Collaborative Support Scheme (ID: 1050648) and funding from the NHMRC Centre of Research Excellence in Medicines and Ageing (CREMA; ID: 1060407). BD is supported by an NHMRC Postgraduate Research Scholarship (ID: 1094325), the Sydney Catalyst Translational Cancer Research Centre and a CREMA PhD scholarship top-up.

Competing interests BEK has received conference support and a speaker's honorarium from Roche.

Ethics approval Population Health Service Research Ethics Committee (approval number: 2010/02/213) and Australian Department of Human Services (DHS) External Review Evaluation Committee (approval numbers: Ml1474, Ml1475 and Ml1477). 
Provenance and peer review Not commissioned; externally peer reviewed.

Open Access This is an Open Access article distributed in accordance with the Creative Commons Attribution Non Commercial (CC BY-NC 4.0) license, which permits others to distribute, remix, adapt, build upon this work noncommercially, and license their derivative works on different terms, provided the original work is properly cited and the use is non-commercial. See: http:// creativecommons.org/licenses/by-nc/4.0/

\section{REFERENCES}

1. Slamon DJ, Clark GM, Wong SG, et al. Human breast cancer: correlation of relapse and survival with amplification of the HER-2/ neu oncogene. Science 1987;235:177-82.

2. Harris CA, Ward RL, Dobbins TA, et al. The efficacy of HER2-targeted agents in metastatic breast cancer: a meta-analysis. Ann Oncol 2011;22:1308-17.

3. Moja L, Tagliabue L, Balduzzi S, et al. Trastuzumab containing regimens for early breast cancer. Cochrane Database Syst Rev 2012;4:CD006243.

4. Balduzzi S, Mantarro S, Guarneri V, et al. Trastuzumab-containing regimens for metastatic breast cancer. Cochrane Database Syst Rev 2014;6:CD006242.

5. Valachis A, Nearchou A, Lind P, et al. Lapatinib, trastuzumab or the combination added to preoperative chemotherapy for breast cancer: a meta-analysis of randomized evidence. Breast Cancer Res Treat 2012;135:655-62.

6. Hicks M, Macrae ER, Abdel-Rasoul M, et al. Neoadjuvant dual HER2-targeted therapy with lapatinib and trastuzumab improves pathologic complete response in patients with early stage HER2-positive breast cancer: a meta-analysis of randomized prospective clinical trials. Oncologist 2015;20:337-43.

7. Sun J, Chen C, Yao X, et al. Lapatinib combined with neoadjuvant paclitaxel-trastuzumab-based chemotherapy in patients with human epidermal growth factor receptor 2-positive breast cancer: a meta-analysis of randomized controlled trials. Oncol Lett 2015;9: 1351-8.

8. Kawalec $\mathrm{P}$, Łopuch $\mathrm{S}$, Mikrut A. Effectiveness of targeted therapy in patients with previously untreated metastatic breast cancer: a systematic review and meta-analysis. Clin Breast Cancer 2015;15:90-100.e1.

9. Broglio KR, Quintana M, Foster M, et al. Association of pathologic complete response to neoadjuvant therapy in HER2-positive breast cancer with long-term outcomes: a meta-analysis. JAMA Oncol 2016;2:751-60

10. Kümler I, Tuxen MK, Nielsen DL. A systematic review of dual targeting in HER2-positive breast cancer. Cancer Treat Rev 2014;40:259-70.

11. Baselga J, Cortés J, Kim SB, et al., CLEOPATRA Study Group. Pertuzumab plus trastuzumab plus docetaxel for metastatic breast cancer. N Engl J Med 2012;366:109-19.

12. Verma S, Miles D, Gianni L, et al. Trastuzumab emtansine for HER2-positive advanced breast cancer. N Engl J Med 2012;367:1783-91.

13. Hutchins LF, Unger JM, Crowley JJ, et al. Underrepresentation of patients 65 years of age or older in cancer-treatment trials. $N$ Engl J Med 1999;341:2061-7.

14. Avorn J. In defense of pharmacoepidemiology-embracing the yin and yang of drug research. N Engl J Med 2007;357:2219-21.

15. Rawlins M. De testimonio: on the evidence for decisions about the use of therapeutic interventions. Lancet 2008;372:2152-61.

16. Banks E, Pearson SA. A life-cycle approach to monitoring benefits and harms of medicines. Med J Aust 2012;197:313-14.

17. Kelman CW, Pearson SA, Day RO, et al. Evaluating medicines: let's use all the evidence. Med J Aust 2007;186:249-52.

18. Kaufman PA, Brufsky AM, Mayer M, et al. Treatment patterns and clinical outcomes in elderly patients with HER2-positive metastatic breast cancer from the registHER observational study. Breast Cancer Res Treat 2012;135:875-83.

19. Tripathy D, Kaufman PA, Brufsky AM, et al. First-line treatment patterns and clinical outcomes in patients with HER2-positive and hormone receptor-positive metastatic breast cancer from registHER. Oncologist 2013;18:501-10.

20. Yardley DA, Kaufman PA, Brufsky A, et al. Treatment patterns and clinical outcomes for patients with de novo versus recurrent HER2-positive metastatic breast cancer. Breast Cancer Res Treat 2014;145:725-34.

21. Yardley DA, Tripathy D, Brufsky AM, et al. Long-term survivor characteristics in HER2-positive metastatic breast cancer from registHER. Br J Cancer 2014;110:2756-64.
22. Jackisch C, Schoenegg W, Reichert D, et al. Trastuzumab in advanced breast cancer-a decade of experience in Germany. BMC Cancer 2014;14:924.

23. Jackisch C, Welslau M, Schoenegg W, et al. Impact of trastuzumab treatment beyond disease progression for advanced/metastatic breast cancer on survival-results from a prospective, observational study in Germany. Breast 2014;23:603-8.

24. Hamy-Petit AS, Belin L, Bonsang-Kitzis $\mathrm{H}$, et al. Pathological complete response and prognosis after neoadjuvant chemotherapy for HER2-positive breast cancers before and after trastuzumab era: results from a real-life cohort. Br J Cancer 2016;114:44-52.

25. Seferina SC, Lobbezoo DJ, de Boer M, et al. Real-life use and effectiveness of adjuvant trastuzumab in early breast cancer patients: a study of the Southeast Netherlands Breast Cancer Consortium. Oncologist 2015;20:856-63.

26. Mustacchi G, Puglisi F, Molino AM, et al. Observational study on adjuvant trastuzumab in HER2-positive early breast cancer patients. Future Oncol 2015;11:1493-500.

27. Ménard S, Balsari A, Tagliabue E, et al., OMERO Group. Biology, prognosis and response to therapy of breast carcinomas according to HER2 score. Ann Oncol 2008;19:1706-12.

28. Dall P, Lenzen G, Göhler T, et al. Trastuzumab in the treatment of elderly patients with early breast cancer: results from an observational study in Germany. J Geriatr Oncol 2015;6: 462-9.

29. Spano J-P, Vignot S, Ho XD, et al. Outcome of HER2-positive breast cancer patients following metastatic relapse after adjuvant trastuzumab treatment since EMA regulatory approval. ASCO Annual Meeting Proceedings, 2012:641.

30. Krell J, James CR, Shah D, et al. Human epidermal growth factor receptor 2-positive breast cancer relapsing post-adjuvant trastuzumab: pattern of recurrence, treatment and outcome. Clin Breast Cancer 2011;11:153-60.

31. Gruschkus S, Doan J, Chen C, et al. First-line patterns of care and outcomes of HER2-positive breast cancer patients who progressed after receiving adjuvant trastuzumab in the outpatient community setting. ASCO Annual Meeting Proceedings, 2010:684.

32. Vaz-Luis I, Seah D, Olson EM, et al. Clinicopathological features among patients with advanced human epidermal growth factor-2-positive breast cancer with prolonged clinical benefit to first-line trastuzumab-based therapy: a retrospective cohort study. Clin Breast Cancer 2013;13:254-63.

33. Marla S, Cardale J, Dodwell DJ, et al. HER2-positive early breast cancers: what proportion are receiving adjuvant trastuzumab therapy? A multicenter audit. J Clin Oncol 2010;28(15 Suppl): 668-8.

34. Vaz-Luis I, Ottesen RA, Hughes ME, et al. Impact of hormone receptor status on patterns of recurrence and clinical outcomes among patients with human epidermal growth factor-2-positive breast cancer in The National Comprehensive Cancer Network: a prospective cohort study. Breast Cancer Res 2012;14:R129.

35. Pearson SA, Ringland CL, Ward RL. Trastuzumab and metastatic breast cancer: trastuzumab use in Australia-monitoring the effect of an expensive medicine access program. $J$ Clin Oncol 2007;25:3688-93.

36. Lu CY, Srasuebkul P, Drew AK, et al. Positive spillover effects of prescribing requirements: increased cardiac testing in patients treated with trastuzumab for HER2+ metastatic breast cancer. Intern Med J 2012;42:1229-35.

37. Lu CY, Srasuebkul P, Drew AK, et al. Trastuzumab therapy in Australia: which patients with HER2+ metastatic breast cancer are assessed for cardiac function? Breast 2013;22:482-7.

38. Parkinson B, Viney R, Haas M, et al. Real-world evidence: a comparison of The Australian Herceptin Program and clinical trials of trastuzumab for HER2-positive metastatic breast cancer. Pharmacoeconomics 2016;34:1039-50.

39. Goldhar HA, Yan AT, Ko DT, et al. The temporal risk of heart failure associated with adjuvant trastuzumab in breast cancer patients: a population study. J Natl Cancer Inst 2016;108(1):1-8.

40. Bonifazi M, Franchi M, Rossi M, et al. Trastuzumab-related cardiotoxicity in early breast cancer: a cohort study. Oncologist 2013;18:795-801.

41. Bonifazi M, Franchi M, Rossi M, et al. Long term survival of HER2-positive early breast cancer treated with trastuzumab-based adjuvant regimen: a large cohort study from clinical practice. Breast 2014;23:573-8.

42. Negri E, Zambelli A, Franchi M, et al. Effectiveness of trastuzumab in first-line HER2 + metastatic breast cancer after failure in adjuvant setting: a controlled cohort study. Oncologist 2014;19:1209-15. 
43. Rossi M, Carioli G, Bonifazi M, et al. Trastuzumab for HER2+ metastatic breast cancer in clinical practice: cardiotoxicity and overall survival. Eur J Cancer 2016;52:41-9.

44. Chavez-MacGregor M, Zhang N, Buchholz TA, et al. Trastuzumab-related cardiotoxicity among older patients with breast cancer. J Clin Oncol 2013;31:4222-8.

45. Shih YC, Xu Y, Dong $W$, et al. First do no harm: population-based study shows non-evidence-based trastuzumab prescription may harm elderly women with breast cancer. Breast Cancer Res Treat 2014;144:417-25.

46. Tsai HT, Isaacs C, Fu AZ, et al. Risk of cardiovascular adverse events from trastuzumab (Herceptin $\left({ }^{(R)}\right)$ ) in elderly persons with breast cancer: a population-based study. Breast Cancer Res Treat 2014;144:163-70.

47. Vaz-Luis I, Keating NL, Lin NU, et al. Duration and toxicity of adjuvant trastuzumab in older patients with early-stage breast cancer: a population-based study. J Clin Oncol 2014;32:927-34.

48. Bowles EJ, Wellman R, Feigelson HS, et al. Risk of heart failure in breast cancer patients after anthracycline and trastuzumab treatment: a retrospective cohort study. J Natl Cancer Inst 2012;104:1293-305.

49. Chen J, Long JB, Hurria A, et al. Incidence of heart failure or cardiomyopathy after adjuvant trastuzumab therapy for breast cancer. J Am Coll Cardiol 2012;60:2504-12.

50. Gallagher CM, More K, Masaquel A, et al. Survival in patients with non-metastatic breast cancer treated with adjuvant trastuzumab in clinical practice. Springerplus 2016;5:395.

51. Vaz-Luis I, Lin NU, Keating NL, et al. Treatment of early-stage human epidermal growth factor 2-positive cancers among medicare enrollees: age and race strongly associated with non-use of trastuzumab. Breast Cancer Res Treat 2016;159:151-62.

52. Gallagher CM, More K, Kamath T, et al. Delay in initiation of adjuvant trastuzumab therapy leads to decreased overall survival and relapse-free survival in patients with HER2-positive non-metastatic breast cancer. Breast Cancer Res Treat 2016;157:145-56.

53. DaCosta Byfield S, Buck PO, Blauer-Peterson C, et al. ReCAP: treatment patterns and cost of care associated with initial therapy among patients diagnosed with operable early-stage human epidermal growth factor receptor 2-overexpressed breast cancer in the United States: a real-world retrospective study. J Oncol Pract 2016;12:159-67.

54. Guérin A, Lalla D, Gauthier G, et al. Comparison of treatment patterns and economic outcomes in metastatic breast cancer patients initiated on trastuzumab versus lapatinib: a retrospective analysis. Springerplus 2014;3:236.

55. Delea TE, Kartashov A, Sharma PP. Retrospective Study of the Prevalence, Predictors, and Consequences of Nonadherence With Lapatinib in Women With Metastatic Breast Cancer Who Were Previously Treated With Trastuzumab. Journal of Pharmacy Technology 2013;30(1):22-30.

56. Pharmaceutical Benefits Advisory Committee 2015 (updated 22 October 2015). http://www.pbs.gov.au/info/industry/listing/ participants/pbac (accessed 5 Apr 2016).

57. Mellish L, Karanges EA, Litchfield MJ, et al. The Australian Pharmaceutical Benefits Scheme data collection: a practical guide for researchers. BMC Res Notes 2015;8:634.

58. Public Summary Document for Trastuzumab, powder for I.V. infusion, 150 mg, Herceptin®, Nov 20082009 (updated 25 March 2009). http://www.pbs.gov.au/info/industry/listing/elements/ pbac-meetings/psd/2008-11/pbac-psd-trastuzumab-nov08 (accessed 5 Apr 2016).

59. Staff. Budget briefs. Sunday Tasmanian. Hobart Town, TAS: News Limited, 2002

60. Cooke G. Govt to make breast cancer treatment drug free of charge. Canberra Times, 13 October 2001;19.

61. Metherell M. Free drug for women with breast cancer. Sydney Morning Herald, 12 October 2001;3.

62. Pharmaceutical Benefits Advisory Committee. Public Summary Document - November 2014 PBAC Meeting. In: Pharmaceutical Benefits Advisory Committee. Australia: Pharmaceutical Benefits Advisory Committee, 2014.

63. Australian Government, Department of Health. Transitioning of Herceptin Subsidy to the Pharmaceutical Benefits Scheme (PBS). http://www.pbs.gov.au/info/news/2015/07/transitioning-ofherceptin-subsidy-to-pbs. Department of Health, 2015.

64. Australian Bureau of Statistics. Australian Standard Geographical Classification (ASGC). Statistical Local Area (SLA). http://www.abs. gov.au/ausstats/abs@.nsf/Lookup/2901.0Chapter23002011 (accessed 5 Apr 2016).

65. Australian Bureau of Statistics. Australian Standard Geographical Classification (ASGC). http://www.abs.gov.au/ websitedbs/D3310114.nsf/home/Australian+Standard+ Geographical+Classification+\%28ASGC\%29 (accessed 5 Apr 2016)

66. Kearny B, Smith M. Response to the Senate Select Committee on Health. In: Parliament of Australia, ed. http://www.aph.gov.au/ DocumentStore.ashx?id=f263db77-ba89-466e-8edb-ae95b880ce72, 2014.

67. Sloan KL, Sales AE, Liu CF, et al. Construction and characteristics of the RxRisk-V: a VA-adapted pharmacy-based case-mix instrument. Med Care 2003;41:761-74.

68. Srasuebkul P, Dobbins TA, Pearson SA, et al., Elements of Cancer Care (EoCC) Investigators. Validation of a proxy for estrogen receptor status in breast cancer patients using dispensing data. Asia Pac J Clin Oncol 2014;10:e63-8.

69. Lu CY, Barratt J, Vitry A, et al. Charlson and Rx-Risk comorbidity indices were predictive of mortality in the Australian health care setting. J Clin Epidemiol 2011;64:223-8.

70. Harris CA, Ward RL, Daniels B, et al. Do pharmaceutical claims accurately reflect oncology prescribing practice? Evidence from an Australian HER2+ early breast cancer cohort (HER2EBC). ASCO Annual Meeting Proceedings, 2013:100.

71. Joshi V, Adelstein B-A, Schaffer A, et al. A Proxy of Cancer Progression in Dispensing Claims: Validation and Performance. Pharmacoepidemiology and Drug Safety: John Wiley \& Sons, 2013:221-22.

72. Young AF, Dobson AJ, Byles JE. Health services research using linked records: who consents and what is the gain? Aust $N Z$ $J$ Public Health 2001;25:417-20.

73. Holman CD. The impracticable nature of consent for research use of linked administrative health records. Aust $N Z J$ Public Health 2001;25:421-2.

74. International Committee of Medical Journal Editors. Recommendations for the Conduct, Reporting, Editing, and Publication of Scholarly Work in Medical Journals 2016 [December 2016: [Available from: http://www.icmje.org/ accessed 15 Jan 2017. 